\title{
Effect of Magnetic Field on Blood Flow in Stenoted Arteries
}

Rotaina J. Essa

rotinajasim@uomosul.edu.iq
Nawara J. Essa

norajassim1987@yahoo.com

College of Computer Sciences and Mathematics

University of Mosul, Mosul, Iraq

Received on: 06/03/2019

Accepted on: 13/05/2019

\section{ABSTRACT}

In this paper, the effect of magnetic field on blood flow in stenoted arteries was studied, stokes equations in polar coordinates were used to describe such flow. All flow characteristics were found under the influence of the magnetic field and how this stenosis affects flow.

Keywords: Magnetic force, blood flow, stenoted arteries.

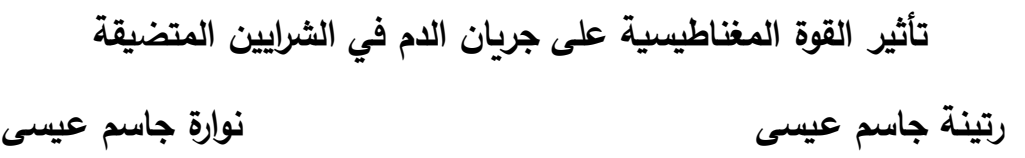

$$
\text { كلية علوم الحاسوب والرياضيات الريات الموصل، الدوصل، العرلق }
$$

تاريخ قبول البحث: $2019 \mid 05 \backslash 13$

تاريخ استلام البحث: $2019 \backslash 03106$

\section{الملخص}

في هذا البحث تمت دراسة تأثير المجال المغناطيسي على جريان الدم في الشرايين المتضيقة. استخدت معادلات ستوكس في الاحداثيات القطبية لوصف هكذا جريان وتم العثور على جميع خصائص التدفق تحت تأثير المجال المغناطيسي وتأثر هذا التدفق بالتضيق. الكلمات المفتاحية: القوة المغناطيسية، جريان الدم، الشرايين المتضيقة.

\section{Introduction 1 المقدمة}

إنَّ دراسة طريقة جريان الدم وتَفقُقه في الشرايين مهمة جداً ؛ لفهم الكثير من أمراض القلب والأوعية الدمويّة

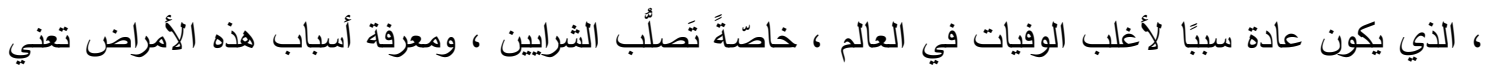

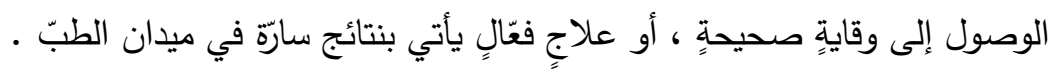

ويمكن التعبير عن جريان الدم وحركته في الشرايين بأنظمة من المعادلات التفاضليّة الجزئيّة غير الخطيّة ،

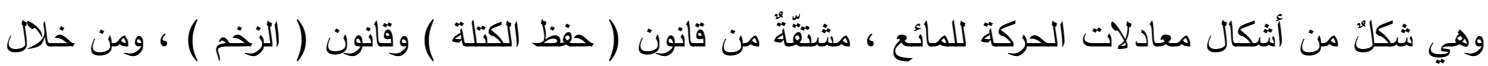
حلّ هذه المعادلات يمكن التعرّف على اضطرابات التدفق في الانحناءات أو التقوسات الثريانية . 
درس عدد من العلماء ، مثل :[3] و[8] تَفْقَُ الدم في الثرايين المتضيقة ، وأثر هذا التضيِّّ في جريان

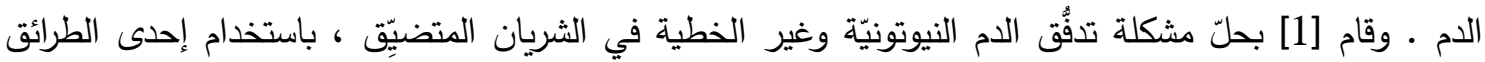
العدديّة . - الع

وقام كلٌّ من الباحتَينِ [2] و [5] بدراسة تدفُق الدم عبر الثرايين ، مع وجود مجالٍ مغناطيسيّ ، وتحت

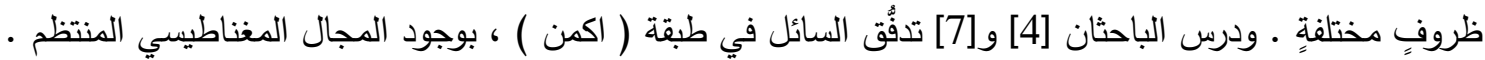
وقام [9] بدراسة تأثير الحقل المغناطيسي في تدفُق الدم في الشرايين ، باستخدام إحدى الطرائق العدديّة أيضًا .

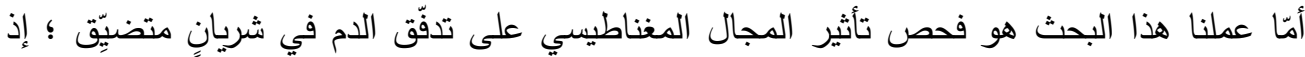

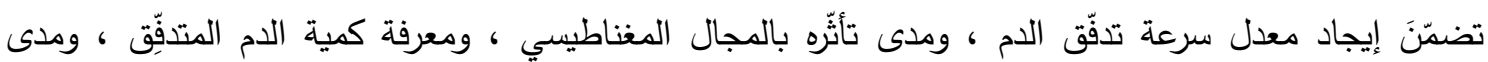

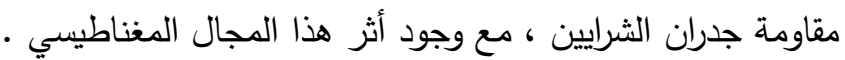
الأنموذج والمعادلات الأساسية في الجريان: ليكن لدينا مقطع لجريان الدم في شريان متضيّق كما في الثكل (1)

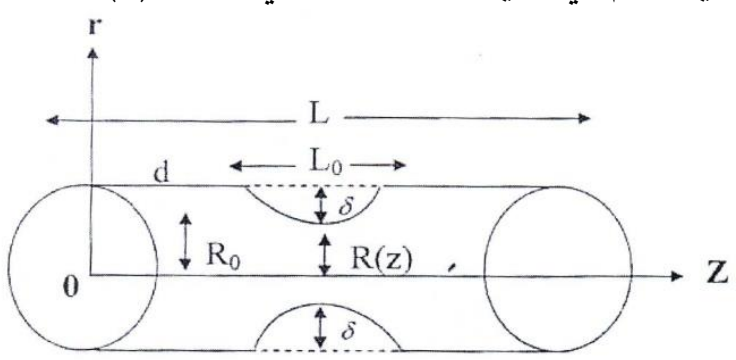

الثكل (1)

إذ إن: - n

R: نصف قطر الثريان و R(z) يمثل نصف قطر منطقة التضيّق في الثريان، L يمثل طول الثريان و يمثل طول منطقة التضيق في الشريان، $\delta$ يمثل أقصى ارتفاع لمنطقة التضيّق، d تحدد المسافة من بداية الثريان إلى منطقة التضيّق. وإن التعبير الرياضي لنصف قطر الثريان يمكن تشثيله ب: $\frac{\mathrm{R}(\mathrm{z})}{\mathrm{R}_{0}}=\left\{\begin{array}{c}1-\mathrm{A}\left[\mathrm{L}_{0}{ }^{(\mathrm{m}-1)}(\mathrm{z}-\mathrm{d})-(\mathrm{z}-\mathrm{d})^{\mathrm{m}}\right] \text { if } \mathrm{d} \leq \mathrm{z} \leq \mathrm{d}+\mathrm{L}_{0} \\ 1\end{array}\right.$

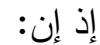

$$
\begin{aligned}
& \mathrm{A}=\frac{\delta}{\mathrm{R}_{0}}\left(\frac{\frac{\mathrm{m}}{\mathrm{m}-1}}{\mathrm{~L}_{0}^{\mathrm{m}}(\mathrm{m}-1)}\right), \mathrm{m} \geq 2 \\
& \frac{\partial \mathrm{p}^{*}}{\partial \mathrm{r}^{*}}=\rho \mathrm{g} \\
& \text { ويمكن تمثيل جريان الدم هذا بالثكل الآتي: }
\end{aligned}
$$




$$
\begin{aligned}
& \lambda=\frac{\Delta \mathrm{p}}{\mathrm{Q}} \\
& \frac{\partial \mathrm{p}^{*}}{\partial \mathrm{z}^{*}}=\frac{\mu}{\mathrm{r}^{*}} \cdot \frac{\partial}{\partial \mathrm{r}^{*}}\left(\mathrm{r} \cdot \frac{\partial \mathrm{w}^{*}}{\partial \mathrm{r}^{*}}\right)-\frac{\sigma \mathrm{B}_{0}^{2}}{\rho} \mathrm{w}^{*}
\end{aligned}
$$

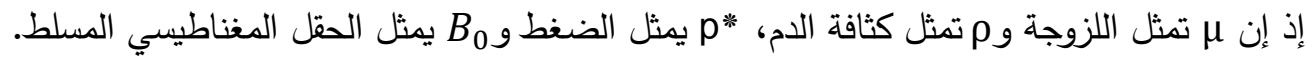
التحليل البعدي: - (البع

لغرض إيجاد المعادلات اللابعدية للمعادلات (1) و (3) يمكن أن نفرض متغيرات لابعدية

$$
\begin{gathered}
\mathrm{r}^{*}=\mathrm{rr}_{0} \quad \mathrm{w}^{*}=\mathrm{ww}_{0} \quad, \quad \mathrm{z}^{*}=\mathrm{zz}_{0} \\
\mathrm{p}^{*}=\mathrm{p} \rho \mathrm{w}_{0}^{2}, \mathrm{~g}^{*}=\mathrm{g}^{2} \mathrm{w}_{0}^{2} \mathrm{~L}
\end{gathered}
$$

وبتعويض هذه القيم اللابعدية في المعادلات (1) و (3) نحصل على معادلات في الحالة اللابعدية.

$$
\begin{aligned}
& \frac{\partial \mathrm{p}}{\partial \mathrm{r}}-\mathrm{kg}=0 \\
& \frac{1}{\mathrm{r}} \frac{\partial}{\partial \mathrm{r}}\left(\mathrm{r} \frac{\partial \mathrm{w}}{\partial \mathrm{r}}\right)-\mathrm{M}^{2} \mathrm{w}=\operatorname{Re} \frac{\partial \mathrm{p}}{\partial \mathrm{z}} .
\end{aligned}
$$

إذ إن Re يمثل عدد رينولد ومعرف على النحو الآتي:

$$
\operatorname{Re}=\frac{\rho \mathrm{r}_{0} \mathrm{w}_{0}}{\mu \mathrm{z}_{0}}
$$

$$
\begin{aligned}
& \text { مع } \\
& \text { ولحل هذا النظام يقديم الشروط الحدودية الآتية: } \\
& \frac{\partial \mathrm{w}}{\partial \mathrm{r}}=0 \quad, \quad \mathrm{r}=0 \\
& \mathrm{w}=0, \quad \mathrm{r}=\mathrm{R}(\mathrm{z}) \\
& \mathrm{p}=\mathrm{p}_{0} \quad, \quad \mathrm{z}=0 \\
& \mathrm{p}=\mathrm{p}_{\mathrm{L}}, \quad \mathrm{z}=\mathrm{L}
\end{aligned}
$$

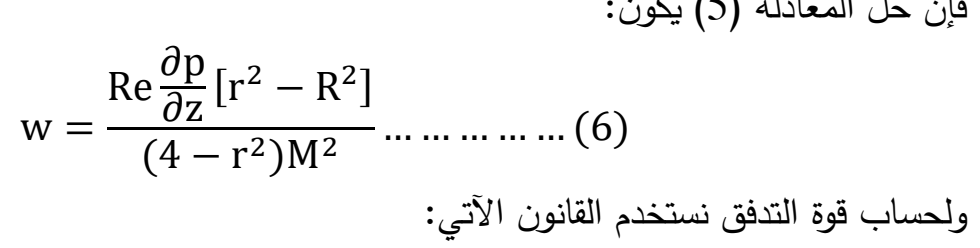

$$
\begin{aligned}
& \text { فإن حل المعادلة (5) يكون: } \\
& Q=\int_{0}^{\mathrm{R}} 2 \pi r w(r) d r
\end{aligned}
$$

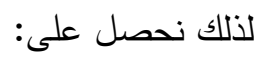

$$
\begin{aligned}
& \mathrm{Q}=\frac{2 \pi \operatorname{Re} \frac{\partial \mathrm{p}}{\partial \mathrm{z}}}{\mathrm{M}^{2}} \int_{0}^{\mathrm{R}} \mathrm{r}\left[\frac{\mathrm{r}^{2}-\mathrm{R}^{2}}{4-\mathrm{r}^{2}}\right] \mathrm{dr}
\end{aligned}
$$




$$
\begin{aligned}
& \mathrm{Q}=\frac{2 \pi R e}{\mathrm{M}^{2}} \cdot \frac{\partial \mathrm{p}}{\partial \mathrm{z}} \int_{0}^{\mathrm{R}} \mathrm{r}\left[1+\frac{4-\mathrm{R}^{2}}{4-\mathrm{r}^{2}}\right] \mathrm{dr} \\
& \text { نكامل المعادلة (9) لنحصل على: } \\
& \mathrm{Q}=\frac{2 \pi R e}{\mathrm{M}^{2}} \cdot \frac{\partial \mathrm{p}}{\partial \mathrm{z}}\left[\frac{\mathrm{R}^{2}}{2}-\frac{1}{2}\left(4-\mathrm{R}^{2}\right) \ln \left|4-\mathrm{R}^{2}\right|+\frac{1}{2} \ln (4)\right] \\
& \mathrm{Q}=\frac{\pi \mathrm{R}_{\mathrm{e}}}{\mathrm{M}^{2}} \frac{\partial \mathrm{p}}{\partial \mathrm{z}} \mathrm{R}^{2} \\
& \frac{\partial \mathrm{p}}{\partial \mathrm{z}}=\frac{\mathrm{M}^{2} \mathrm{Q}}{\pi \operatorname{Re} \mathrm{R}^{2}} \\
& \int_{0}^{\mathrm{L}} \partial \mathrm{p}=\frac{\mathrm{M}^{2} \mathrm{Q}}{\pi \operatorname{Re}} \int_{0}^{\mathrm{L}}\left(\frac{1}{\left(\frac{\mathrm{R}(\mathrm{z})}{\mathrm{R}_{0}}\right)^{2}}\right) \partial \mathrm{z} \\
& \mathrm{p}_{\mathrm{L}}-\mathrm{p}_{0}=\frac{\mathrm{M}^{2} \mathrm{Q}}{\pi \mathrm{Re}} \mathrm{F}(\mathrm{z}) \\
& \mathrm{F}(\mathrm{z})=\int_{0}^{\mathrm{L}} \frac{1}{\left(\frac{\mathrm{R}(\mathrm{z})}{\mathrm{R}_{0}}\right)^{2}} \mathrm{dz} \ldots \ldots \ldots \ldots
\end{aligned}
$$$$
\text { ويمكن تبسيط المعادلة (10) لتصبح: }
$$

ومن معادلة (11)

والآن نكامل المعادلة (12)

ويمكن كتابة المعادلة (15) بالثكل:

$$
\begin{gathered}
F(z)=\int_{0}^{d} \frac{1}{\left(\frac{R(z)}{R_{0}}\right)^{2}} d z+\int_{d}^{d+L_{0}} \frac{1}{\left(\frac{R(z)}{R_{0}}\right)^{2}} d z+\int_{d+L_{0}\left(\frac{R(z)}{R_{0}}\right)^{2}}^{d} \frac{1}{L} \ldots \ldots(16) \\
F(z)=L-L_{0}+\int_{d}^{d} \frac{1}{\left[1-A_{0}\left(L_{0}^{m-1}(z-d)-(z-d)^{m}\right]^{2}\right.} d z \ldots \ldots \\
p_{L}-p_{0}=\frac{M^{2} Q}{\pi R e}\left[L-L_{0}+\int_{d}^{d+L_{0}} \frac{1}{\left[1-A\left(L_{0}^{m-1}(z-d)-(z-d)^{m}\right]^{2}\right.} d z\right] \ldots
\end{gathered}
$$
ولحساب مقاومة التدفق , فإن :

$$
\lambda=\frac{\Delta \mathrm{p}}{\mathrm{Q}}=\frac{\mathrm{p}_{\mathrm{L}}-\mathrm{p}_{0}}{\mathrm{Q}}
$$

$$
\lambda_{0}=\frac{\frac{M^{2} \mathrm{Q}}{\pi R_{0}}\left[L-L_{0}+\int_{d}^{d+L_{0}} \frac{1}{\left[1-A\left(L_{0}^{m-1}(z-d)-(z-d)^{m}\right]^{2}\right.} d z\right]}{Q}
$$




$$
\lambda_{0}=\frac{\mathrm{M}^{2}}{\pi \operatorname{Re}}\left[\mathrm{L}-\mathrm{L}_{0}+\int_{\mathrm{d}}^{\mathrm{d}+\mathrm{L}_{0}} \frac{1}{\left[1-\mathrm{A}\left(\mathrm{L}_{0}^{\mathrm{m}-1}(\mathrm{z}-\mathrm{d})-(\mathrm{z}-\mathrm{d})^{\mathrm{m}}\right]^{2}\right.} \mathrm{dz}\right] \ldots \ldots
$$

إذن معادلة (21) تصبح بالثكل:

$\lambda_{0}$

$=\frac{\mathrm{M}^{2}}{\pi \mathrm{R}_{\mathrm{e}}}\left[\mathrm{L}-\mathrm{L}_{0}\right.$

$\left.+\int_{d}^{d+L_{0}} \frac{1}{\left[1-\frac{8}{R_{0}}\left(\frac{m^{\frac{m}{m-1}}}{L_{0}^{m}(m-1)}\right)\left(L_{0}^{m-1}(z-d)-(z-d)^{m}\right)\right]^{2}} d z\right]$

وبحل المعادلات (6) و (22) باستخدام نظام (Matlab) يمكن ملاحظة تأثير المجال المغناطيسي في خصائص التدفق .كما في الثكلين (2) و(3).

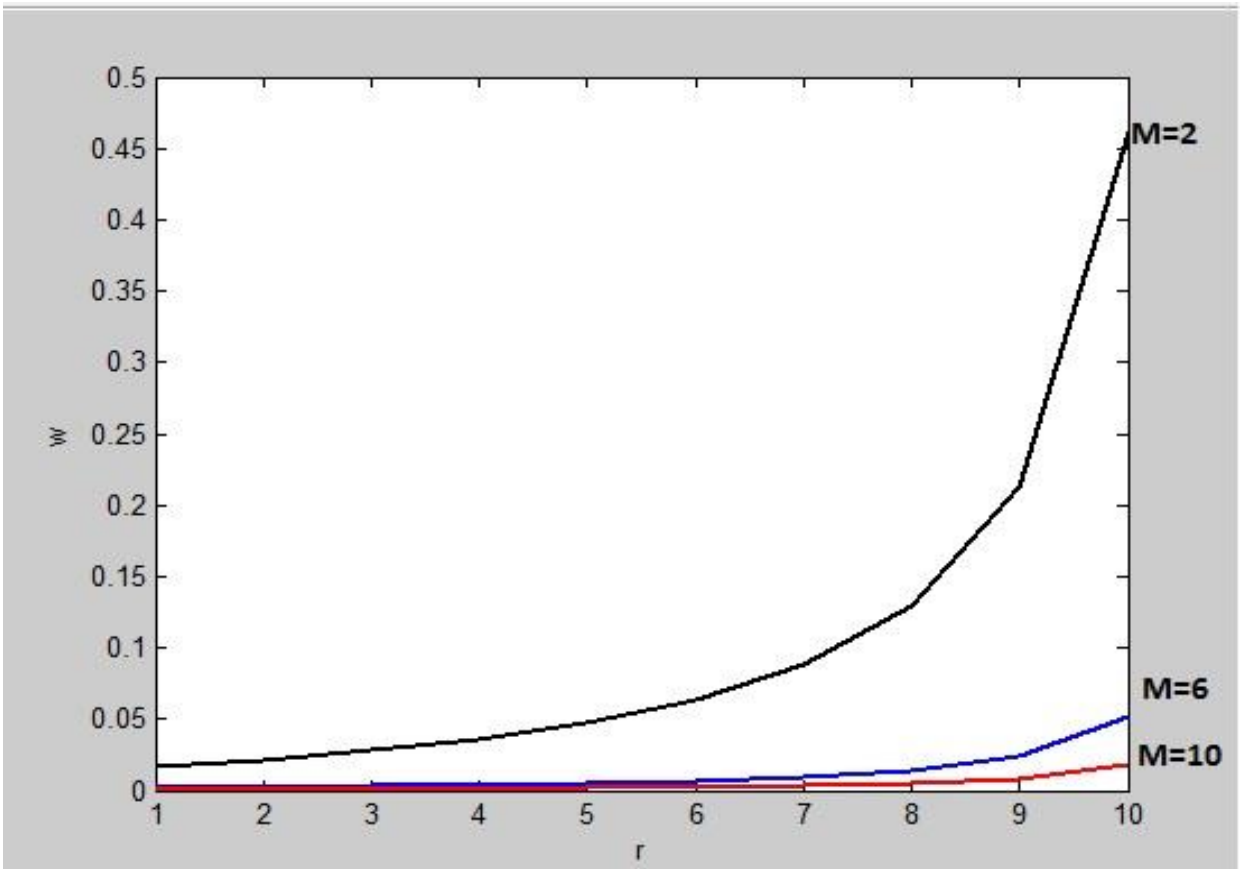

الثكل (2.2) يوضح سرعة تدفق الدم بتغير قيمة القوة المغناطيسية

الثكل (2.2) يوضح العلاقة بين القوى المغناطيسية M و سرعة تدفق الدم W, حيث نلاحظ كلما زادت القوى المغناطيسية فإن سرعة تدفق الدم تقّل. 


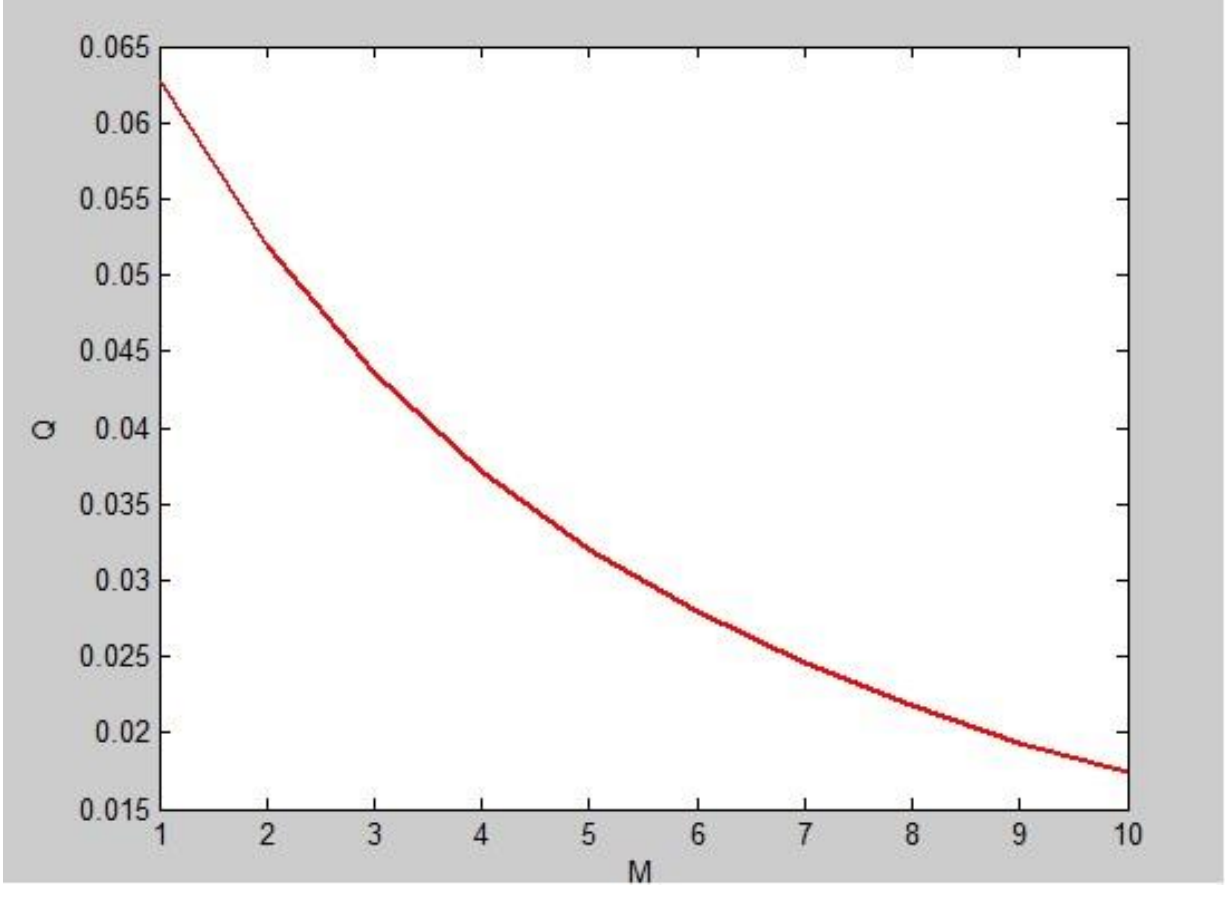

الثكل (2.3) يبين العلاقة بين التدفق والقوة المغناطيسية

الثكل (2.3) يوضح العلاقة بين القوى المغناطيسية M و قوة التدفق Q, حيث نلاحظ كلما ازداد المجال المغناطيسي يؤدي إلى قلة التدفق.

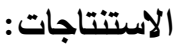

في هذا البحث قمنا بحساب تأثير المجال المغناطيسي في خصائص الدم مثل سرعة الدم والتدفق ومقاومة

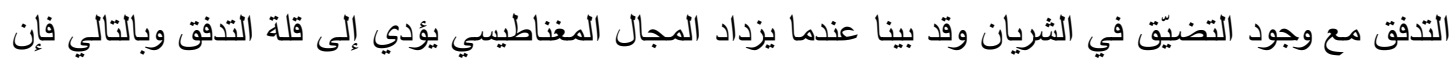
سرعة الدم تقل مما يؤدي إلى زيادة المقاومة. وقد يكون هذا مفيداً للأطباء الممارسين لعلاج مرضى ارتفاع ضغط الدم ومرضى تصلب الثرايين من خلال المجال المغناطيسي. 


\section{المصاد}

[1] Chakravarty, S., \& Sen, S. (2006). A mathematical model of blood flow and convective diffusion processes in constricted bifurcated arteries. Korea-Australia Rheology Journal, 18(2), 51-65.

[2] Milnor W.R, Hemodynamic (2nd ed.), William and Wilkins, Baltimore, 1989.

[3] Mishra, B. K., \& Verma, N. (2010). Effect of stenosis on non-Newtonian flow of blood in blood vessels. Australian Journal of Basic and Applied Sciences, 4(4), 588-601.

[4] Misra, J. C., Pal, B., \& Gupta, A. S. (1998). Hydromagnetic flow of a second-grade fluid in a channel-Some applications to physiological systems. Mathematical Models and Methods in Applied Sciences, 8(08), 1323-1342.

[5] Misra, J. C., Pal, B., Pal, A., \& Gupta, A. S. (2001). Oscillatory entry flow in a plane channel with pulsating walls. International journal of non-linear mechanics, 36(5), 731-741.

[6] Pal, B., Misra, J. C., \& Gupta, A. S. (1996). Steady hydromagnetic flow in a slowly varying channel. Proceedings-National Academy of Sciences, India. Section A, Physical Sciences, 66(pt. 3), 247-262.

[7] Pavlov, K. B. (1974). Magnetohydrodynamic flow of an incompressible viscous fluid caused by deformation of a plane surface. Magnitnaya Gidrodinamika, 4(1), 146-147.

[8] Sapna. Ratan Shah and S.V. Siddiqui, (July-August 2011), "Acomprative Study for the non_nwetonian behavior of blood flow through atheros_clerotic arterial segment", Vol.9, Issu.2, Article-019.

[9] Varshney, G., Katiyar, V., \& Kumar, S. (2010). Effect of magnetic field on the blood flow in artery having multiple stenosis: a numerical study. International Journal of Engineering, Science and Technology, 2(2), 96782 . 